\title{
Tin in Early Chinese Ceramics
}

\author{
Blythe McCarthy*
}

*Freer Gallery of Art and Arthur M. Sackler Gallery, Smithsonian Institution, Washington DC 20560

Several Chinese ceramics with shapes characteristic of bronze vessels and covered with the remains of a tin foil cladding have been found in Hunan province. Attributed to the eastern Zhou period (720-221 B.C.) and associated with the Ch'u culture these vessels are believed to have been manufactured in imitation of either bronze or silver objects. Three objects of this type were studied, including one $h u$ or large vase, and two $d u i$, or pair of footed bowls that sit one on top of another to form a closed oval shape. The ceramics have a gray body with scattered small fragments of tin foil remaining on the $h u$ and one $d u i$ and areas of white accretion on the second dui. A yellow to brown adhesive material used to attach the tin to the ceramic was found on all of the objects (FIG. 1).

Non-destructive XRF analyses of the surface of the tin foil identified small amounts of tin, copper, iron, zinc and lead. However, only tin was seen in EDS analyses of uncorroded areas of a polished cross-section of the foil (FIG. 2). Corroded areas were found to contain tin and oxygen, agreeing with previous analyses of similar objects that found the foil to be a very pure tin. Antimony and sulfur were also found by the previous investigators who suggested that the tin was treated with antimony, arsenic or sulfur to form a colored surface layer, thus imitating bronze [1]. No antimony was found in the studies described here and although EDS analysis of the surface did reveal a high concentration of sulfur, it is primarily associated with organic materials on the surface. The organic material was found on both surfaces of the samples of foil studied. A thin white surface layer on the second $d u i$ was determined to be cassiterite ( $\mathrm{SnO} 2)$ using $\mathrm{x}$-ray diffraction, confirming the former presence of a tin layer. While tin is fairly stable under normal atmospheric conditions, it is susceptible to degradation when exposed to moisture and few objects of tin or with tin embellishments have been found from ancient China.

Tin was used in China in large amounts as a component of bronze [2] and is also often found in small amounts in copper containing glasses and ceramic glazes, where its presence is generally ascribed to tin found in ores of those metals or to the use of recycled bronze as a raw material [3]. No examples of tin oxide opacification are currently known among ancient Chinese glasses and glazes, however, tin oxide inclusions were found in some turquoise enamels that covered areas of iron pigment in a group of Cizhou-type bulb jars from the Yuan period (1279-1368 A.D.) [4].

\section{References}

[1] I. Newton, Far Eastern Ceramics Bulletin 10 (1958) 3.

[2] R.J. Gettens, The Freer Chinese Bronzes, Technical Studies II, Smithsonian Institution, Washington DC, 1969.

[3] N. Wood, Chinese Glazes, Their Origins, Chemistry and Recreation, U. of Pennsylvania Press, Philadelphia, 1999.

[4] B. McCarthy et al. in Proceedings of the 2002 International Symposium on Ancient Ceramics Its Scientific and Technological Insights Chinese Academy of Science, Shanghai, 2002. 


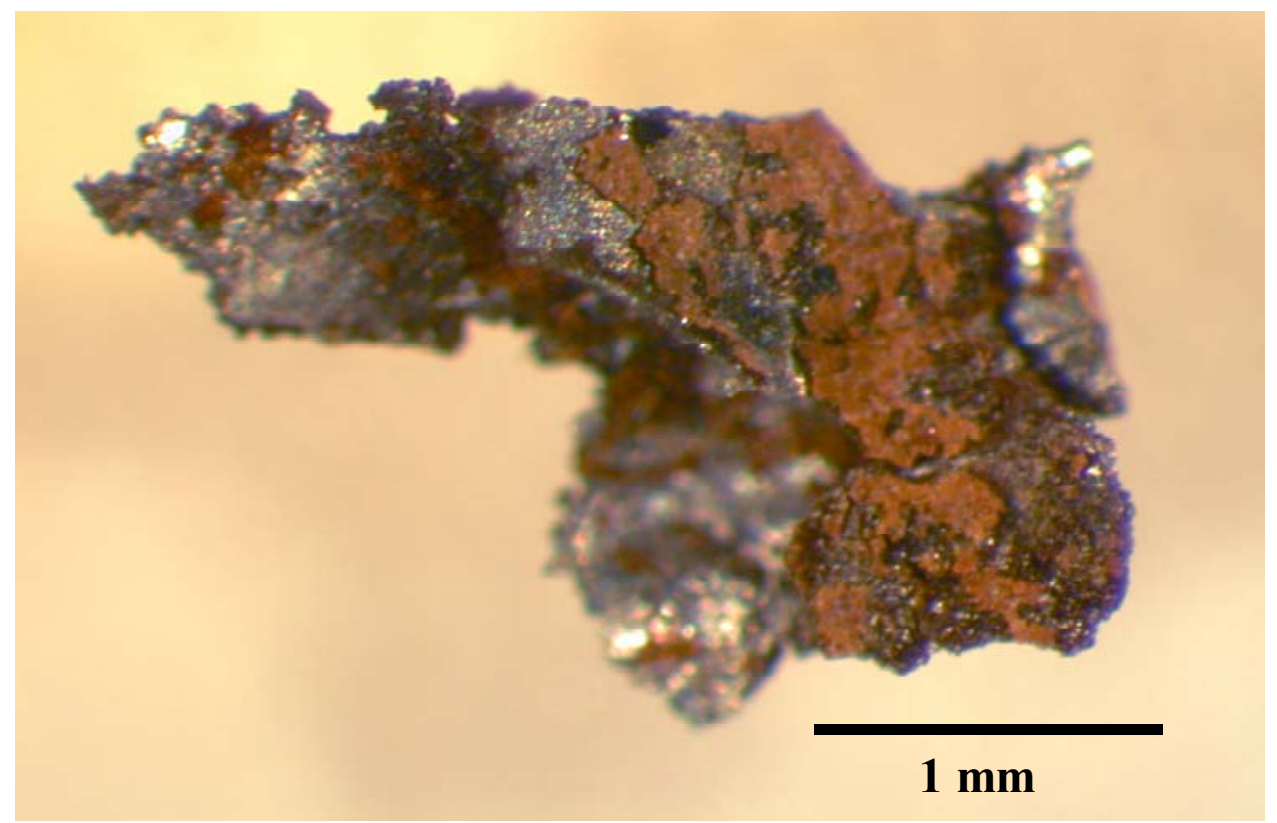

FIG. 1. Tin foil from RLS1997.48.1870 folded at right to show upper (right) and lower (left) surfaces of the foil. The brown areas are organic material, an adhesive.

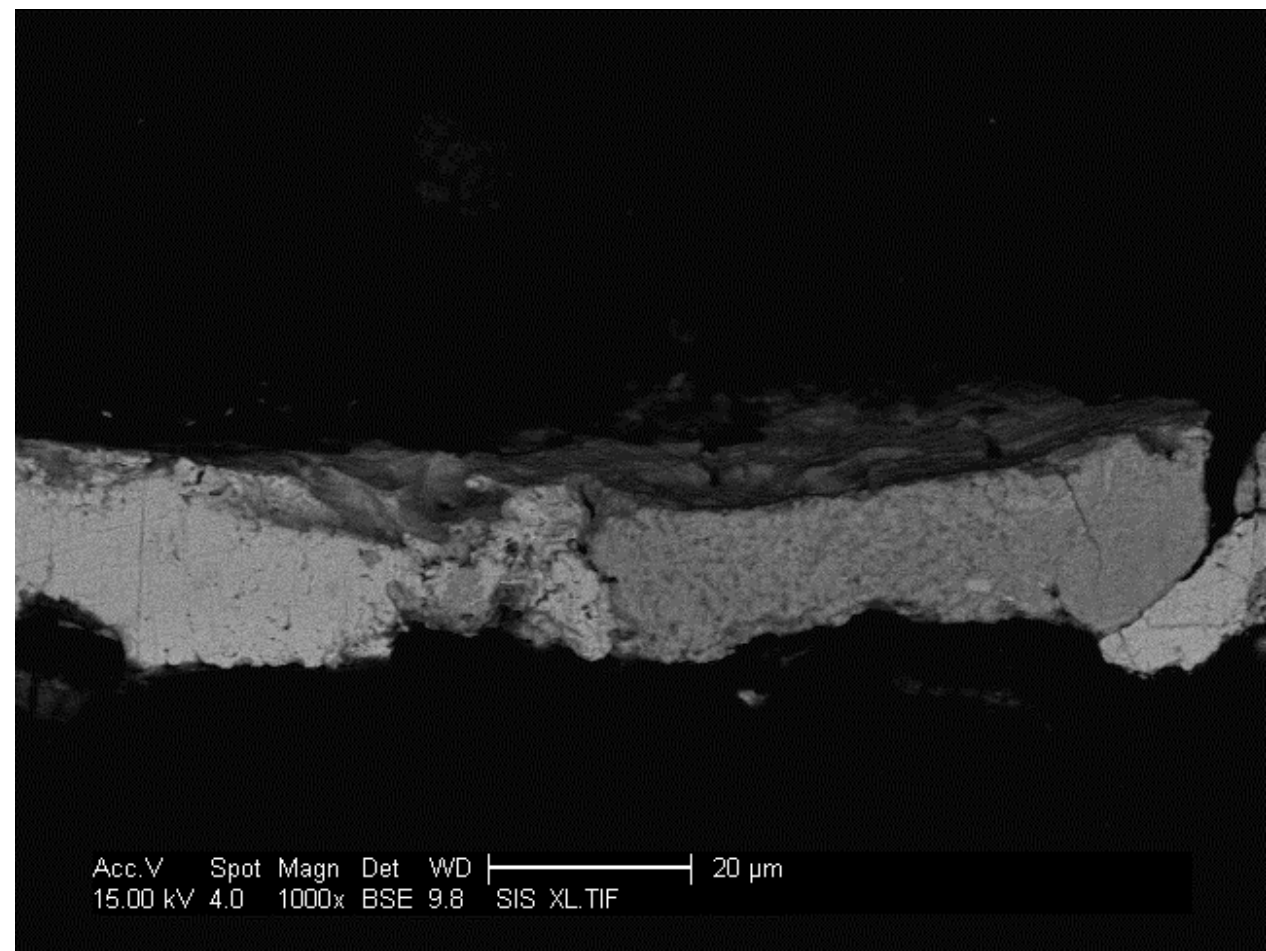

FIG. 2. Backscattered electron image of a polished cross-section of tin foil from RLS1997.48.1912 showing tin metal (bright areas) and tin oxide (dark areas). Mounting material was epoxy (black). 